\title{
Progress on the Pest Risk Analysis of China
}

\author{
Jianwei Li ${ }^{1}$, Xiaoyan Zhang ${ }^{2, *}$, Yibing Liang ${ }^{1}$, Hao $\mathrm{Wu}^{1}$ \\ ${ }^{1}$ General Administration of Quality Supervision, Inspection and Quarantine of China, Beijing 100088, \\ China \\ ${ }^{2}$ Fujian Entry-Exit Inspection and Quarantine Bureau of China, Fuzhou 350001, China
}

\author{
我国进境植物检疫风险分析进展 \\ 李建伟 ${ }^{1}$, 张晓燕 ${ }^{2, *}$, 梁忆冰 ${ }^{1}$, 吴昊 ${ }^{1}$ \\ 1 国家质量监督检验检疫总局，北京 $100088 ， 中$ 国 \\ ${ }^{2}$ 福建出入境检验检疫局, 福州 350001 , 中国
}

\begin{abstract}
In this paper, a comprehensive review of the entry pest risk analysis of China was conducted on five aspects, such as legal framework, organization structure and management procedures, technical methods, information management, and application of risk analysis. It can be concluded that the pest risk analysis (PRA) has been widely used and become the basis for developing the policies and measures of the import and export plant quarantine. After years of practice, the legal framework, the organization structure, the management procedures and the technology methods of PRA were initially established in China. A great deal of PRA reports had been finished. Information management system were also established. The recommendations related to how to improve the entry pest risk analysis were also made in this paper.
\end{abstract}

Keywords: entry, phytosanitary, risk analysis, progress

\section{摘要}

本文从法律制度、组织架构和工作程序、风 险分析应用、技术方法以及辅助工具研究等五个 方面, 对我国进境植物检疫风险分析现状进行了 全面的综述。认为有害生物风险分析已经成为制

*通讯作者: E-mail: zhangxy8668@126.com
定进出境植物检疫政策和措施的基础, 在我国进 出境植物检疫领域得到了广泛应用。经过多年实 践, 我国已经建立了较为完善的风险分析的法律 制度、组织架构和工作程序以及风险评估技术体 系, 组织开展了大量的有害生物风险评估实践活 动, 在辅助工具的开发方面也取得了重要进展。 本文还就如何完善进境植物检疫风险分析工作提 出了相关建议。

关键词: 进境; 植物检疫; 风险分析; 进展

1 前言

风险分析的目的在于确定不利事件对作用对 象的影响程度 ${ }^{[1]}$ 。进境植物检疫的核心任务是防 止外来重要植物有害生物传入, 其本质就是对外 来植物有害生物传入的风险进行科学分析和评

估, 以确定采取措施的力度。

早期的进境植物检疫对危险“对象”采取的是 全面禁止进口的“零风险”管理措施, 但世界贸易 组织(简称 WTO)乌拉圭回合谈判中达成的《实施 卫生与植物卫生措施协定》(简称《SPS 协定》), 明确要求各缔约成员通过科学评估承担“可接受 的检疫风险”, 并保证各项卫生与植物卫生措施 (简称 SPS 措施)建立在有害生物风险分析的基础 之上, 从而将 SPS 措施对贸易的影响降到最低限 度 ${ }^{[2]}$, 因此, 《SPS 协定》推动了进出境植物检疫 从“零风险”向“可接受风险”转变, 管理从管“对象” 向管“风险”转变, 同时推动了风险分析在进境植 物检疫领域的应用。为协调 WTO 各成员植物检 疫风险分析工作, 1995 年以来, WTO 承认的三 


\section{Risk Analysis and Crisis Response in Big Data Era (RAC-16)}

个国际组织之一的国际植物保护公约（简称 IPPC) 秘书处, 陆续发布了 4 项与植物检疫风险 分析有关的国际植物检疫措施标准(简称 ISPMs), 这些国际标准成为各国遵从的规则和标准。

有害生物风险分析工作分为开始、风险评估 和风险管理三个阶段。根据 ISPM No.5《植物检 疫术语表》[3]，有害生物风险是指: “有害生物传 入和扩散的可能性及潜在的经济影响程度。”有害 生物风险分析(Pest Risk Analysis, 简称 PRA)是指: “运用生物学、其他科学及经济方面的证据进行评 估，以确定某种生物体是否为有害生物，是否应 被限定以及应采取的植物检疫措施的力度。”有害 生物风险评估是指: “评价有害生物传入和扩散的 可能性及有关潜在经济影响的程度”。有害生物风 险管理是指: “评价和选择减少有害生物传入和扩 散的风险方案”。

\section{2 我国进境植物检疫风险分析工作进展}

根据《SPS 协定》, 有害生物风险分析已成为 世界各国制定植物检疫技术法规、政策和采取植 物检疫措施的基础。自上世纪 90 年代中国引入有 害生物风险分析以来, 风险分析已成为进境植物 检疫政策、措施制定的重要依据。经过多年的探 索实践, 我国已经建立了较为完善的风险分析的 法律制度、组织架构和工作程序, 组织开展了大 量的有害生物风险分析实践活动，在风险评估技 术方法、辅助工具的开发方面也取得了重要进展, 为促进我国农产品国际贸易健康发展做出了积极 贡献。

\section{1 风险分析法律制度建设现状}

我国植物检疫的主要法律依据是《中华人民 共和国进出境动植物检疫法》及其实施条例。该 法颁布于 1991 年, 当时风险分析的概念尚未被广 泛接受, 所以未明确写入该法及其实施条例之中。 2001 年 12 月 11 日, 中国正式成为 WTO 成员。 作为成员国必须遵循相关国际规则, 因此, 《SPS 协定》以及国际植物检疫措施标准在中国得到了 较好地执行, 并成为当前中国进出境植物检疫和 风险分析的重要依据。

在部门规章方面, 2001 年, 国家质量监督检 验检疫总局（简称国家质检总局）发布了《出入 境检验检疫风险预警及快速反应管理规定》，使用
了《SPS 协定》中的“风险评估”、“预警”等概念, 标志着我国开始建立基于风险评估的检验检疫风 险预警及快速反应机制。2002 年, 国家质检总局 发布了《进境植物和植物产品风险分析管理规 定》。该规定参照《SPS 协定》和 ISPMs 的有关原 则和要求, 对风险分析启动、风险评估和风险管 理的相关内容、程序或要求都做出了明确的规定。 这两个规章连同相关国际规则和标准仍然是当前 进出境植物检疫有害生物风险分析工作的主要法 律依据。

\section{2 风险分析组织架构和工作程序现状}

\subsection{1 风险分析组织构架}

目前，我国农业、林业、环保等相关部门都 从各自角度分别建立了风险评估体系。在进出境 植物检疫领域, 主要由国家质检总局牟头组织开 展有害生物风险分析。

1991 年, 农业部原植物检疫实验所开始探索 进行有害生物风险分析。1995 年 5 月, 农业部原 进出境动植物检疫局成立了“中国植物有害生物 风险分析工作组”, 正式将有害生物风险分析应用 于中国进境植物检疫工作实践, 给进出境植物检 疫带来了新的管理理念 ${ }^{[4]}$ 。2000 年, 原国家出入 境检验检疫局在动植物检疫实验所正式设立了 “PRA 办公室”, 这是我国第一个专门的 PRA 机构 [5]。2002 年 4 月, 国家质检总局牵头成立了跨部 门的“中国进出境动植物检验检疫风险分析委员 会”，委员会由相关政府主管部门领导、科研院校 和检验检疫系统知名专家等共同组成, 对重大植 物检疫管理措施和科学决策进行技术咨询和指 导。

根据 2002 年发布的《进境植物和植物产品风 险分析管理规定》, 现有的风险分析组织架构和职 责是：国家质检总局负责风险分析启动与最终风 险管理措施制定和对外发布; 动植物检疫实验所 风险分析办公室负责具体风险分析工作技术标准 的制定和风险分析报告的审核; 全国 35 个直属检 验检疫局和相关直属事业单位承接具体风险分析 任务。

为更加科学和有效管理有害生物风险分析工 作, 国家质检总局成立了由各专业专家组成的全 国进出境植物检疫风险分析专家库, 参与相关风 险分析报告的审核和技术指导。经过多年工作实 践和积累, 培养和锻炼了一支有害生物风险分析 
Risk Analysis and Crisis Response in Big Data Era (RAC-16)

技术队伍, 有力支撑了进出境植物检疫风险分析 的开展。

\subsection{2 风险分析工作程序}

根据《进境植物和植物产品风险分析管理规 定》, 风险分析工作主要分为以下 6 个步骤: 第一 步, 由国家质检总局启动风险分析，并下达风险 分析任务; 第二步, 承接单位组织开展具体的风 险分析，提交包含风险管理措施方案在内的风险 分析报告; 第三步, 由风险分析办公室负责组织 审核风险分析报告，其中特别重要的风险分析报 告还要交由中国进出境动植物检疫风险分析委员 会审议; 第四步, 国家质检总局审定风险管理措 施方案, 征求有关部门、行业、企业及 WTO 成 员意见; 第五步, 国家质检总局完成必要的法律 程序后对外发布风险管理措施, 并通报 WTO; 第 六步, 相关风险管理措施以具有法律管理效力的 书面文件发布出入境检验检疫系统执行 ${ }^{[4]}$ 。

\section{3 有害生物风险分析应用现状}

ISPM No.11《检疫性有害生物风险分析(包括 环境风险和活体转基因生物分析)》将有害生物风 险分析分为以有害生物为起点、以传播途径为起 点和以管理政策措施修订为起点, 这些不同起点 的有害生物风险分析在我国进境植物检疫领域都 得到了不同程度的实践与应用。

\subsection{1 以传播途径为起点的有害生物风险分析}

这类 PRA 主要是针对某种/某类农产品或运 输方式为传播途径可能传带有害生物的风险进行 分析。以传播途径为起点的 PRA 在进出境植物检 疫领域应用非常广泛。1993 年, 原农业部植物检疫 实验所主持完成我国第一个 PRA 报告《对美国(华 盛顿州、加利福尼亚州)地中海实蝇的危险性分 析》, 为美国苹果顺利输华提供科学依据。此后, 国家质检总局每年都开展数十项农产品检疫准入 谈判, 先后解决了多项从有害生物发生国家有条 件地进口我国需要的农产品的植物检疫技术问 题。这些农产品国际贸易的顺利开展均是基于有 害生物风险分析所提出的风险管理措施方案。仅 2014-2015 年, 中国检验检疫科学研究院就对有输 华意向的土耳其樱桃、墨西哥黑莓和树莓、加拿 大蓝莓、澳大利亚油桃、智利油桃、俄罗斯燕麦 和大麦、蒙古水飞蓟、阿根廷踠豆开展了有害生 物风险分析。以传播途径为起点的 PRA 在当前风 险分析工作中数量最多。

\subsection{2 以有害生物为起点的有害生物风险分析}

这类 PRA 一般对已发现或重要有害生物, 评 估它能引起新的入侵, 或继续扩散传播的风险。

其应用重点之一是对某种外来有害生物在中 国适生区进行分析预测。它在我国进境植物检疫 特定管理名单制定过程中发挥了重要作用。如, 上世纪 80-90 年代, 原农业部植物检疫实验所为 制定《进口植物检疫对象名单》、《禁止进口植物 名单》 ${ }^{[4]}$ 、《进境植物检疫危险性病虫杂草名录》、 《进境植物检疫禁止进境物名录》、《进境植物检 疫潜在危险性病虫杂草名录》和修订《进境植物 检疫禁止进境物名录》所做的有害生物风险分析。 2007 年修订颁布新的《进境植物检疫性有害生物 名录》, 更是集合、凝聚了国家质检总局、农业部、 国家林业局, 以及全国多所高校和科研院所数十 位专家多年来有害生物风险分析工作成果。近年 来, 为了控制有害生物的扩散或者分析有害生物 造成的经济影响, 相关专家开展了稻水象甲 ${ }^{[6]}$ 、 检疫性实蝇 ${ }^{[7]}$ 、紫茎泽兰 ${ }^{[8]}$ 、刺若龙葵 ${ }^{[9]}$ 等以有害 生物为起点的风险分析。

其应用重点之二是当境外发生重大植物疫 情, 或者我国口岸截获重要外来危险有害生物, 已有植物检疫措施不足以有效控制风险时, 国家 质检总局参照国际规则和标准, 组织专家在风险 分析基础上, 以警示通报或紧急通知形式发出风 险预警, 采取紧急措施或强化措施。例如, 2000 年 1 月 1 日起对美国、日本等输华货物的木质包 装采取紧急措施和 2001 年 7 月 1 日起对进口原木 实施新的植物检疫措施, 分别是基于《松材线虫 随美国和日本输华货物木质包装材料传入中国的 风险分析报告》和《关于中国从国外进口原木截 获有害生物的风险评估报告》。近年来, 为防止油 菜茎基溃疡病等检疫性有害生物的传入, 在风险 分析基础上, 国家质检总局 2009 年发布了《关于 进口油菜籽实施紧急检疫措施公告》, 要求行政相 对人完善监管设施、加强疫情监测, 并组织开展 了进口油菜籽监测检测、热处理、定量风险评估 等研究。

\subsection{3 以管理政策措施修订为起点的有害生物风} 险分析

除上述两类有害生物风险分析外, 当外部条 件发生改变, 需要重新调整修订原有植物检疫措 施时, 国家质检总局也会启动开展相关风险分析 工作。例如, 在总结以前工作实践和开展相关引 


\section{Risk Analysis and Crisis Response in Big Data Era (RAC-16)}

进物植物检疫风险评估的基础上，国家质检总局 缩小了检疫审批的范围, 2014 年 10 月 28 日起, 干制失活的植物、昆虫标本等三类科研展览用引 进物不再需要植物检疫审批。又如, 应秘鲁官方 申请, 经中秘双方技术碰商和专家评估, 中方同 意对秘鲁输华柑橘在原有两种冷处理指标 $\left(11^{\circ} \mathrm{C}\right.$ 以下连续处理 15 天或 $1.67^{\circ} \mathrm{C}$ 以下连续处理 17 天) 上增加 $2.22^{\circ} \mathrm{C}$ 以下连续处理 21 天的冷处理指标。

\section{4 风险评估技术方法研究现状}

风险评估属于有害生物风险分析的第二个 阶段, 其工作内容的重点包括对有害生物传入、 扩散的可能性和潜在经济影响的分析。风险评估 多采用定性分析、定量分析，以及定性与定量结 合的方法进行。在多年实践基础上，在国家质检 总局组织下, 先后发布了《进出境植物和植物产 品有害生物风险分析技术要求》( GB/T 20879-2007)、《进出境植物和植物产品有害生物 风险分析工作指南》(GB/T 21658-2008)、《有害生 物风险分析框架》 $(\mathrm{GB} / \mathrm{T}$ 27617-2011)等多个国家 标准, 以及《进出境植物和植物产品风险分析技 术要求》(SN/T1601.1-2005)、《进出境植物和植 物产品有害生物风险分析程序》(SN/T 1601.2-2005)、《杂草风险分析技术要求》 （SN/T1893-2007）等多个行业标准, 基本构建了 我国的有害生物风险分析技术体系。该体系目前 主要以传播途径为起点、以定性方法为主要评估 方法。

\subsection{1 定性分析方法}

定性分析方法最常用的是使用非概率抽样方 法将风险事件分解成若干风险要素, 建立评价风 险因素的指标体系和评判标准，然后按照一定规 则对各个风险要素的评价值通过加权、几何平均 或其他的多维向量运算法则等进行综合评估, 即 得出最终的评价。

上世纪 90 年代, 原农业部植物检疫实验所主 持农业部“八五”重点课题“PRA 研究”, 开展了有 关风险分析评价指标体系和多指标综合评判分析 方法研究, 提出的多指标综合评价法及其量化指 标评估体系 ${ }^{[10]}$ 得到了广泛应用, 接近当时国际先 进水平。此后, 很多学者运用各种评判方法不断 尝试建立新的评估方法和指标体系, 如周国梁 (2006)等利用层次分析法, 提出了基于传播途径的 外来有害生物风险分析的分级标准和评价方法
${ }^{[11]}$; 孙楠(2008)等提出了我国进口水果果实有害 生物风险分析评价指标和分析方法 ${ }^{[12]}$; 葛卜峰 (2015)等建立了进境船舶压舱水监管风险评估模 型和指标体系 ${ }^{[13]}$ 。采取多指标综合评判的定量分 析, 提高了定性分析的量化程度。

\subsection{2 定量分析方法}

风险评估定量分析方法是在时间和空间上 分析造成风险的各个风险事件(场景分析), 用数学 语言来描述这些风险事件, 建立其间的函数关系 (数学模型), 并对其进行计算机模拟, 最终结果多 用风险发生的概率估计来表述。

定量分析使用的数学方法和模型很多, 诸如 物种适生区的建模与预测, 有害生物生长发育模 型, 物种入侵、定殖过程中种群动态过程以及物 种传播与扩散的相关模型等等。在定量分析时, 要考虑具体的数据特性, 然后再选择较为适宜的 统计方法 ${ }^{[14]}$ 。

定量风险分析一般都要借助工具软件来进 行。2000 年, 中美专家开展了小麦矮腥黑穗病菌 (TCK) 定量风险分析的合作研究, 利用场景分析和 蒙物卡罗方法对 TCK 进入麦田的可能性进行计 算, 探索寻找小麦种子携带 TCK 孢子的安全数 量。这是我国第一个真正的定量有害生物风险评 估 ${ }^{[15]}$ 。此后, 我国在定量风险分析方面做了大量 的研究和探索。例如, 李志红(2007)等采用 CLIMEX 地点比较模型运作与 ArcGIS 插值分析 相结合的方法, 明确了 42 种实蝇在我国的适生范 围和适生程度 ${ }^{[16]}$ 。石娟用灰色一马尔科夫链模型 对松材线虫入侵对马尾松林分生长的影响进行了 预测 ${ }^{[17]}$ 。卢辉(2012)等应用 MaxEnt 预测了木薯单 爪螨在全球的潜在地理分布 ${ }^{[18]}$ 。武威(2015)等应 用“CLIMEX+GIS”的方法, 对黑森瘞蚊在中国的 适生区进行了分析预测 ${ }^{[19]}$ 。

2012 年, 中国农业大学李志红教授等与澳大 利亚和美国专家合作, 运用地理、气象、寄主、 有害生物地理分布、有害生物检疫截获基础数据, 利用多种技术和工具, 分别从入侵、定殖、定殖 扩散范围、经济损失等方面进行了较为系统的有 害生物风险定量分析技术研究。例如利用 SOM+Matlab 对 180 种经济实蝇有害生物风险进 行初篮, 评估有害生物的定殖可能性 ${ }^{[20]}$; 利用场 景模型 $+@$ RISK, 对番石榴果实蝇随寄主果实传 入我国可能性进行评估预测 ${ }^{[21]}$; 利用经济损失模 型+@RISK, 对南亚果实蝇对我国南瓜产业的潜 
在经济损失进行评估 ${ }^{[22]}$ 。

由于有害生物风险的复杂性和不确定性均 很高, 研究采用定性与定量相结合的方法, 逐步 提高有害生物风险分析的量化水平，有助于提高 风险的可比性, 也有助于风险管理者更清楚地认 识风险的大小, 制定更科学的风险管理措施。

\section{5 有害生物风险分析的辅助工具开发现状}

从 1981 年起, 农业部原植物检疫实验所曾收 集整理了世界各地 6841 种有害生物的寄主范围、 传播途径、危害程度、国内外分布及国外检疫情 况等有关资料，1985 年后又开发建立了《植物有 害生物信息检索系统》。1999 年王峻等(1999)建立 了《进境植物检疫有害生物图文信息检索系统》 [23], 这些信息系统先后为有害生物风险分析工作 提供了信息支持。

2005 年, 国家质检总局开始启动《中国国家 有害生物检疫信息系统》的研发和建设, 2012 年 获国家计算机软件著作权授权后正式运行并进入 互联网。该数据库建有有害生物信息查询、进境 检疫要求信息、风险评估应用、口岸应用信息多 个子系统, 含 4.7 万有害生物和 5.8 万寄主植物等 相关信息, 总数据量达 100 万以上。系统不仅可 以承担风险分析任务有关有害生物名单篮选和辅 助风险评估模型应用, 更重要的是为进出境风险 分析工作提供了有力信息支持。

\section{3 关于进境植物检疫风险分析的建议}

\section{1 进一步完善有害生物风险分析法律制度建设}

虽然《SPS 协定》及 ISPMs 已在我国得到了 较好的遵循和执行, 有害生物风险分析也得到了 广泛的应用, 但到目前为止, 在国内相关法律层 面都没有明确提及风险分析, 仅在部门规章层面 对有害生物分析工作进行了规定，有害生物风险 分析的法律效力偏低。因此, 建议在法律层级明 确风险分析的地位, 以保证风险分析更广泛、有 效地应用于进境植物检疫风险管理中。

\section{2 强化管理, 促进风险分析工作科学高效运转}

一是加强顶层设计, 对进出境植物检疫风险 分析的运行管理和技术研究进行系统规划, 全面 提升风险分析水平; 二是加强“风险分析办公室”
在各个风险分析任务中的组织协调; 三是组织开 展风险分析方法和关键技术研究; 四是加强风险 分析的技术交流。充分利用系统内外专家资源和 “中国进出境动植物检验检疫风险分析委员会”工 作机制, 更好地培养风险分析技术队伍。

\section{3 开展更加系统深入的风险分析技术研究}

一是成立进境植物检疫风险国家级中心实验 室, 专门从事有害生物风险分析技术、方法研究 和验证; 二是加强国际标准和国外先进做法研究, 重新评估和审视我国植物检疫风险分析国家标准 和行业标准, 完善风险分析技术体系; 三是加强 定量有害生物风险评估等基础性研究工作, 为以 传播途径为起点的风险评估工作奠定更坚实的基 础; 三是加强评估方法和技术的研究。在大数据 基础上, 探索利用智联网等工具, 集合大范围专 家学者智慧, 进一步提升有害生物风险分析的科 学性。在有害生物风险评估指标中, 指标的量化 存在不确定性, 评估结果等级评判也存在不确定 性, 采用云模型可以处理定性分析中广泛存在的 随机性和模糊性, 实现定性概念和定量数值之间 不确定性的转换, 从而提高 PRA 数据处理的客观 性 ${ }^{[24]}$; 四是加强我国进境植物检疫风险可接受水 平的研究, 为风险分析技术方法的确定奠定基础。

\section{参考文献}

[1] 牟瑞芳. 系统风险理论的基本问题. 风险分 析和危机反应的创新理论和方法一《中国灾害 防御协会风险分析委员会第五届年会论文 集》.中国灾害防御协会风险分析专业委员会, 2012 年, P45-49.

[2] 葛志荣. 《实施卫生与植物卫生措施协定》的 理解. 中国农业出版社, 2001 年, 北京.

[3] 黄冠胜主编. 国际植物检疫措施标准.中国标 准出版社, 北京, 2010 年, P120.

[4] 蒲民, 吴杏霞, 梁忆冰, 等. 论我国进境植物 检疫风险分析体系的构建. 植物检疫, 2009, 23(5): 44-47.

[5] 陈洪俊, 范晓虹, 李蔚民. 我国有害生物风险 分析(PRA)的历史与现状. 植物检疫, 2002, 16(1):28-32.

[6] 齐国君, 高燕, 黄德超, 等.基于 MAXENT 
Risk Analysis and Crisis Response in Big Data Era (RAC-16)

的稻水象甲在中国的入侵扩散动态及适生性 分析.植物保护学报, 2012, 39(2):129-136.

[7] 马兴莉, 李志红, 胡学难,等.橘小实蝇、瓜实 蝇和南亚果实蝇对广东省造成的经济损失评 估.植物检疫, 2013，27(3):50-56.

[8] 方炎, 秦萌, 李志红, 等.紫茎泽兰对我国花 生产业造成的潜在经济损失评估.中国农业大 学学报, 2015, 20(6):146-151.

[9] 方志刚, 方炎, 秦萌, 等.刺薷龙葵对中国玉 米产业造成的潜在经济损失评估.中国农业大 学学报, 2015, 20(6):138-145.

[10] 蒋青,梁忆冰,王乃扬, 等.有害生物危险性评价 的定量分析方法研究. 植物检疫, 1995, 9(4): 208-211.

[11] 周国梁, 印丽萍, 黄晓藻.外来有害生物风险 分析指标体系的建立. 植物检疫, 2006, 8(20): 14-19.

[12] 孙楠, 黄冠胜, 李志红, 等.进口水果果实有害生 物风险分析实用方法的建立. 植物检疫, 2008, 22(1): 27-31.

[13] 葛卜峰, 高晓清, 刀彩华.进境船舶压舱水检 疫监管风险评估指标体系的建立. 植物检疫, 2015, 29(4): 26-30.

[14] 张秀玲, 黄英, 朱水芳, 等.入侵生物适生区 与种群动态中的数学方法. 植物检疫, 2015 , 29(2): 7-14.

[15] 姚文国, 章正, 黄冠胜主编.小麦矮化腥黑穗 病.中国农业出版社, 北京, 2002 .

[16]李志红. 生物入侵防控: 重要经济实蝇潜在地 理分布研究.中国农业大学出版社 2015 年 北 京: P1.

[17]石娟, 骆有庆, 武海卫.松材线虫 Bursaphelenchus xylophilus 入侵对马尾松
Pinus massoniana 林分生长的影响及相关生 长模型.生态学报，2008，28(7):3193-3204.

[18]卢辉, 陈青, 卢芙萍, 等. 木薯单爪螨全球潜 在地理分布的 MaxEnt 预测. 植物检疫, 2012, 26(1):1-6.

[19]武威, 李志红, 杭小溪.基于 CLIMEX 的黑森 瘦蚊在我国的潜在适生区预测. 植物检疫, 2015, 29(1): 20-24.

[20] Yujia Qin, et al. Global Establishment Risk of Economically Important Fruit Fly Species (Tephritidae). PLOS ONE, Christian Wegener, University of Wurzburg, Germany, January 14, 2015, 1-8.

[21] Xingli Ma, et al. Using Decision Tools Suite to Estimate the Probability of the Introcuction of Bactrocera correcta(Bezzi) Into China via imported Host Fruit. Sensor Letters, Vol.10, 586-591, 2012.

[22]方炎, 李志红, 秦萌, 等. 南亚果实蝇对我国 南瓜产业的潜在经济损失评估. 植物检疫, 2015, 29(3): 28-33.

[23]王峻,林朝森,何钦显, 等.进境植物检疫有害生 物图文信息检索系统. 植物检疫, 1999, 13(1): 56-58.

[24] Y. He. Assessment Research of Bijie Drought Risk Based On Cloud Model. Journal of Risk Analysis and Crisis Response, 2013, 3(4): 192-200. 